\title{
Kinematic Modeling and Analysis of Skid-Steered Mobile Robots With Applications to Low-Cost Inertial-Measurement-Unit-Based Motion Estimation
}

\author{
Jingang Yi, Senior Member, IEEE, Hongpeng Wang, Member, IEEE, Junjie Zhang, Student Member, IEEE, \\ Dezhen Song, Member, IEEE, Suhada Jayasuriya, Senior Member, IEEE, and Jingtai Liu, Member, IEEE
}

\begin{abstract}
Skid-steered mobile robots are widely used because of their simple mechanism and high reliability. Understanding the kinematics and dynamics of such a robotic platform is, however, challenging due to the complex wheel/ground interactions and kinematic constraints. In this paper, we develop a kinematic modeling scheme to analyze the skid-steered mobile robot. Based on the analysis of the kinematics of the skid-steered mobile robot, we reveal the underlying geometric and kinematic relationships between the wheel slips and locations of the instantaneous rotation centers. As an application example, we also present how to utilize the modeling and analysis for robot positioning and wheel slip estimation using only low-cost strapdown inertial measurement units. The robot positioning and wheel slip-estimation scheme is based on an extended Kalman filter (EKF) design that incorporates the kinematic constraints for accuracy enhancement. The performance of the EKF-based positioning and wheel slip-estimation scheme are also presented. The estimation methodology is tested and validated experimentally on a robotic test bed.
\end{abstract}

Index Terms-Extended Kalman filter (EKF), mobile robots, motion estimation, slip estimation, skid steering.

\section{INTRODUCTION}

$\mathbf{S}$ KID steering is a widely used locomotion mechanism for mobile robots. For a skid-steered robot, such as the one shown in Fig. 1, there is no steering mechanism, and motion direction is changed by turning the left- and right-side wheels at different velocities. This mechanism design makes the robot mechanically robust and simple for outdoor navigation. Due to

Manuscript received February 13, 2009; revised May 31, 2009. First published July 28, 2009; current version published October 9, 2009. This paper was recommended for publication by Associate Editor K. Iagnemma and Editor J.-P. Laumond upon evaluation of the reviewers' comments. The work of J. Yi was supported in part by the National Science Foundation under Grant CMMI-0856095. The work of $\mathrm{H}$. Wang was supported by a Fellowship from the Chinese Scholarship Council. This paper was presented in part at the 2007 IEEE/RSJ International Conference on Intelligent Robots and Systems, San Diego, CA, October 29-November 2, 2007, and the 2009 IEEE International Conference on Robots and Automation, Kobe, Japan, May 12-17, 2009.

J. Yi is with the Department of Mechanical and Aerospace Engineering, Rutgers University, Piscataway, NJ 08854 USA (e-mail: jgyi@ rutgers.edu).

H. Wang and J. Liu are with the Institute of Robotics and Automation Information Systems, Nankai University, Tianjing 300071, China (e-mail: wanghp@ robot.nankai.edu.cn; liujt@ robot.nankai.edu.cn).

J. Zhang and S. Jayasuriya are with the Department of Mechanical Engineering, Texas A\&M University, College Station, TX 77843 USA (e-mail: jjzhang@tamu.edu; sjayasuriya@tamu.edu).

D. Song is with the Department of Computer Science and Engineering, Texas A\&M University, College Station, TX 77843 USA (e-mail: dzsong@cse.tamu.edu).

Color versions of one or more of the figures in this paper are available online at http://ieeexplore.ieee.org.

Digital Object Identifier 10.1109/TRO.2009.2026506

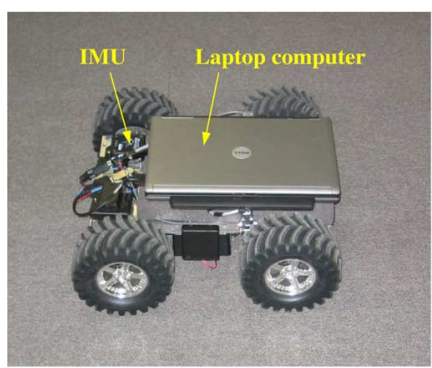

(a)

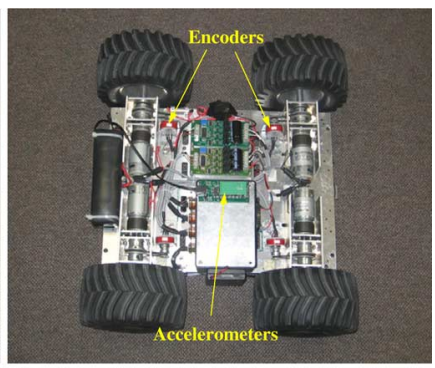

(b)
Fig. 1. Skid-steered four-wheel mobile robot with a sensor suite.

complex wheel/ground interactions and kinematic constraints, it remains a challenging task to obtain an accurate kinematic and dynamic model for skid-steered mobile robots.

Wheel slip plays a critical role in kinematic and dynamic modeling of skid-steered mobile robots. The slip information provides a connection between the wheel rotation velocity and the linear motion of the robot platform. Understanding the slip information is important for robot localization applications, such as dead reckoning. The accuracy of the dead-reckoning technique deteriorates when wheel slip is not negligible. For example, for outdoor terrain navigation robots, such as Mars Exploration Rovers, large wheel slips have been observed [1], [2]. The wheel slip information also plays an important role in robot dynamics and control. The wheel/ground interactions directly provide traction and braking forces that affect the motion stability and maneuverability. The characteristics of the wheel/ground interaction greatly depend on the wheel slip.

The focus of this paper is to analyze the kinematics of skid-steered mobile robots and to apply the analytical results to robot motion estimation. For a four-wheel skid-steered robot (see Fig. 1), we utilize the modeling techniques for tracked vehicles/robots and analyze the geometric and kinematic relationships between wheel slips and instantaneous rotation centers of the robot platform. As an application example of the modeling and analysis, we also present an inertial measurement units (IMUs) based positioning and wheel slip-estimation scheme for skid-steered mobile robots. The motion-estimation scheme is based on the measurements of a low-cost strapdown IMU and wheel encoders. We design an extended Kalman filter (EKF) with three velocity constraints and estimates that are obtained from kinematic modeling and analysis. The proposed positioning and wheel slip-estimation methods are experimentally tested and validated. 
The main contribution of this study is that the new kinematic modeling and analysis of the skid-steered robot reveal the underlying geometric and overconstrained kinematic relationship between the wheel slip and the instantaneous rotation centers. The kinematic modeling and analysis provide "virtual" velocity estimates and constraints of the skid-steered robots. Integration of these "virtual" velocity measurements with the onboard lowcost IMU and wheel encoders improves the motion-estimation accuracy significantly. The robot positioning and wheel slip estimation scheme has shown a superior performance than those results obtained without incorporating the newly developed velocity estimates. The modeling and analysis approach can be further used for robot stability control and motion planning [3].

The remainder of this paper is organized as follows. We start reviewing some related work in Section II. In Section III, we discuss the kinematic modeling of a four-wheel skid-steered mobile robot and present the robot velocity estimates and constraints. Section IV presents the EKF-based robot positioning and wheel slip-estimation design. Experimental results are presented in Section $\mathrm{V}$ before we conclude the paper and discuss future work.

\section{RELATED WORK}

Modeling and control of differential-driven mobile robots, such as unicycle robots, have been extensively studied over the past two decades. The nonholonomic constraint of zero lateral velocity of the wheel contact points is normally considered for differential-driven mobile robots without omniwheels. However, for skid-steered mobile robots, nonzero wheel lateral velocity is allowed, and the zero-velocity constraint is no longer valid. When the wheels on each side of a skid-steered robot are running at the same velocity, the robot actually behaves similar to tracked vehicles. Because of this similarity, methods of modeling tracked ground vehicles have been utilized to model skid-steered mobile robots. For example, Song et al. [4] use the kinematics of tracked vehicles in [5] to estimate wheel slip by a sliding-mode observer design. Because of the difficulty to accurately capture the skid-steering models, in [6] and [7], an extra wheeled trailer is designed to experimentally study the kinematic relationship for simultaneous localization and mapping (SLAM) applications. It is concluded that a kinematic model for an ideal differential-driven wheeled robot cannot be used for skid-steered robots. In [8] and [9], geometric analogy with an ideal differential-driven wheeled mobile robot is studied, and experimental validations have been conducted for both tracked vehicles and skid-steered mobile robots.

In [10], a comparison study is presented for the control performance of an omnidirectional mobile robot with and without considering the wheel slip. It is found that the magnitude of the wheel slip increases as the wheel/ground friction coefficient increases. In [11], wheel skidding and slipping are modeled as disturbances, and control systems designs are presented for wheeled mobile robots under skidding and slipping effects. In [12], a slip-based adaptive trajectory tracking control system is designed for skid-steered robots assuming that wheel slips are obtained in real time.
In localization applications, dead reckoning is one of the major techniques. However, the accuracy of the dead-reckoning technique deteriorates when wheel slip is not negligible. Global positioning system (GPS) also has drawbacks of signal robustness, availability, and accuracy issues. Using inertial devices, such as an IMU, to localize a mobile robot has been studied during the last decade. It is well known that directly integrating acceleration measurements to obtain position information is problematic since any bias or noise measurements in acceleration signals will "blow off" the estimation quickly.

In [13] and [14], the error reduction of the odometry of skidsteered robots is discussed for dead-reckoning applications using encoder and motor current measurement information. Endo et al. [15] discuss a slip compensation odometry scheme for a skid-steered tracked vehicle. A kinematics model of a unicycle robot is used, and an empirical slip kinematics relationship is employed to enhance the localization accuracy. In [8] and [9], the localization of a tracked vehicle is presented based on kinematic models provided in [16]-[18]. In [19], an IMU-based wheel slip-detection scheme is designed for a unicycle-like mobile robot using a dynamic-model-based EKF design with or without GPS measurements. In [2], wheel slip is compensated in an EKF-based localization design with vision odometry. The EKF-based slip compensation scheme in [20] only considers the lateral slip into the kinematics model for an articulated mobile robot, and a laser scanner is also used to provide orientation information with fixed known landmarks. In [21] and [22], velocity constraints, such as zero lateral velocity, are integrated with an EKF or information filter to enhance the navigation accuracy of an autonomous ground vehicle. The EKF design in [21] and [22] utilizes GPS and wheel-encoder measurements, but wheel slip is not considered.

We have incorporated the wheel slip information into the kinematics model of skid-steered robots [23]. An EKF-based motion-estimation design has been also presented in [23] with some preliminary experimental results. In [3], we extend the kinematics modeling and analysis in [23] and provide a dynamic model analysis for skid motion stability. This paper is an extension of our previous results in [3] and [23]. We here provide not only a detailed description of the kinematic modeling and analysis of skid-steered robots but also performance analysis and comprehensive experimental validations of the EKF-based robot positioning and wheel slip-estimation design.

\section{RobOT KINEMATIC MODELS}

\section{A. Kinematics Relationship}

Fig. 2 shows the kinematics schematic of a skid-steered robot. Without loss of generality, we consider the following assumptions: 1) The mass center of the robot is located at the geometric center of the body frame; 2) each side's two wheels rotate at the same speed; and 3) the robot is running on a firm ground surface, and four wheels are always in contact with the ground surface.

Let $\omega_{i}$ and $v_{i}, i=1, \ldots, 4$ denote the wheel angular and center linear velocities for front-left, rear-left, front-right, and rear-right wheels, respectively. From the aforementioned 


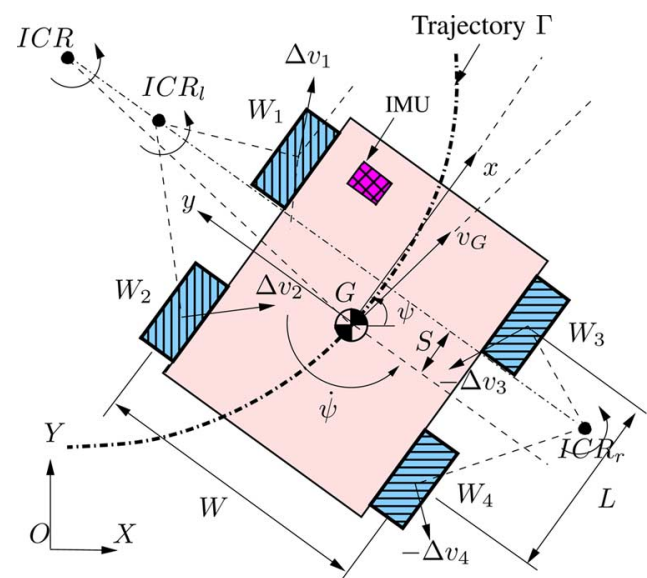

Fig. 2. Top-view schematic of a skid-steered mobile robot.

assumption, we have

$$
\omega_{L}:=\omega_{1}=\omega_{2}, \quad \omega_{R}:=\omega_{3}=\omega_{4} .
$$

We define an inertial frame $\mathcal{I}(X, Y, Z)$ and a robot body frame $\mathcal{B}(x, y, z)$, as shown in Fig. 2. We use Euler angles to describe the transformation between frames $\mathcal{B}$ and $\mathcal{I}$. Let $\Theta:=\left[\begin{array}{lll}\phi & \theta & \psi\end{array}\right]^{T}$ denote the attitude angles, i.e., the roll angle $\phi$ (around $x$-axis), pitch angle $\theta$ (around $y$-axis), and yaw angle $\psi$ (around $z$-axis), respectively. The transformation from $\mathcal{I}$ to $\mathcal{B}$ is considered as the $Z-Y-X$ ordered Euler angle rotation. It is straightforward to calculate the transformation relationship from frames $\mathcal{B}$ to $\mathcal{I}$ given by the following matrix:

$$
\begin{aligned}
C_{B}^{I} & :=C_{B}^{I}(\phi, \theta, \psi) \\
& =\left[\begin{array}{ccc}
c_{\theta} c_{\psi} & -s_{\psi} c_{\phi}+c_{\psi} s_{\phi} s_{\theta} & s_{\phi} s_{\psi}+c_{\psi} s_{\theta} c_{\phi} \\
c_{\theta} s_{\psi} & c_{\phi} c_{\psi}+s_{\theta} s_{\phi} s_{\psi} & -s_{\phi} c_{\psi}+s_{\theta} c_{\phi} s_{\psi} \\
-s_{\theta} & c_{\theta} s_{\phi} & c_{\phi} c_{\theta}
\end{array}\right]
\end{aligned}
$$

where $c_{\theta}:=\cos \theta, \quad s_{\theta}:=\sin \theta$, and the same notation convention is used for the angles $\phi$ and $\psi$.

Let $L$ and $W$ denote the longitudinal and lateral wheel bases, respectively, as illustrated in Fig. 2. The velocity of the robot mass center is denoted by $v_{G}$. Given velocity $v_{G}$ in frame $\mathcal{B}$ and yaw rate $\dot{\psi}$, the wheel center velocities along the $x$-axis for leftand right-side's wheels are given by

$$
\begin{aligned}
& v_{L x}:=v_{1 x}=v_{2 x}=v_{G x}-\frac{W}{2} \dot{\psi} \\
& v_{R x}:=v_{3 x}=v_{4 x}=v_{G x}+\frac{W}{2} \dot{\psi} .
\end{aligned}
$$

For the IMU located at $\left(x_{M}, y_{M}, 0\right)$ in $\mathcal{B}$, we obtain the IMU velocity $\mathbf{v}_{B}=\left[\begin{array}{lll}v_{B x} & v_{B y} & v_{B z}\end{array}\right]^{T}$ in $\mathcal{B}$ as

$$
v_{B x}=v_{G x}-y_{M} \dot{\psi}, \quad v_{B y}=v_{G y}+x_{M} \dot{\psi} .
$$

We define the longitudinal wheel slip $\lambda_{i}$ at each wheel as the ratio of the wheel velocity and its center velocity, namely

$$
\lambda_{i}:=\frac{r \omega_{i}-v_{i x}}{r \omega_{i}}=-\frac{\Delta v_{i x}}{r \omega_{i}}, \quad i=1, \ldots, 4
$$

where $r$ is the effective wheel radius, and $\Delta v_{i x}:=v_{i x}-r \omega_{i}$. For left- and right-side's wheels, the wheel slips satisfy

$$
\lambda_{L}:=\lambda_{1}=\lambda_{2}, \quad \lambda_{R}:=\lambda_{3}=\lambda_{4}
$$

due to the robot operating assumption. It is also observed that wheel slip $\lambda \in[0,1]$ if the wheel is under traction and $\lambda \in(-\infty, 0]$ if the wheel is under braking. In order to constrain the slip calculation within the magnitude of one, using the same treatment as in [16], we take $\lambda=-1$ if $\lambda<-1$ under the braking case. Therefore, we consider the wheel slip $\lambda \in[-1,1]$.

Let $\mathrm{ICR}_{l}, \mathrm{ICR}_{r}$, and $\mathrm{ICR}_{G}$ denote the instantaneous center of rotation (ICR) of the left-side wheel contact points, rightside wheel contact points, and the robot body, respectively. It is known that $\mathrm{ICR}_{l}, \mathrm{ICR}_{r}$, and $\mathrm{ICR}_{G}$ lie on a line parallel to the $y$-axis [8], [18], [24]. We denote the coordinates for $\mathrm{ICR}_{l}, \mathrm{ICR}_{r}$, and $\mathrm{ICR}_{G}$ in $\mathcal{B}$ as $\left(x_{l c}, y_{l c}, 0\right),\left(x_{r c}, y_{r c}, 0\right)$, and $\left(x_{G c}, y_{G c}, 0\right)$, respectively.

Definition 1: We define the longitudinal ICR location, which is denoted as $S$, as the $x$-coordinate of the collinear $\mathrm{ICR}_{l}, \mathrm{ICR}_{r}$, and $\mathrm{ICR}_{G}$ in frame $\mathcal{B}$.

From the aforementioned discussion, the longitudinal ICR location $S$ (see Fig. 2) satisfies the following constraints [8], [24]:

$$
S=x_{l c}=x_{r c}=x_{G c}=-\frac{v_{G y}}{\dot{\psi}} .
$$

We write the longitudinal slip velocities of the wheel/ground contact points as

$$
\begin{aligned}
& \Delta v_{1 x}=\Delta v_{2 x}=\left(y_{l c}-\frac{W}{2}\right) \dot{\psi} \\
& \Delta v_{3 x}=\Delta v_{4 x}=\left(y_{r c}+\frac{W}{2}\right) \dot{\psi} .
\end{aligned}
$$

If the robot turns counterclockwise, as shown in Fig. 2, we have $\Delta v_{1 x}=\Delta v_{2 x} \geq 0$ and $\Delta v_{3 x}=\Delta v_{4 x} \leq 0$, and this implies that the left-side's two wheels are under braking and the right-side's two wheels are under traction. Combining (4) and (8), for $\dot{\psi} \neq 0$, we obtain

$$
\begin{aligned}
y_{l c} & =\frac{v_{1 x}-r \omega_{1}}{\dot{\psi}} \quad y_{r c}=\frac{v_{3 x}-r \omega_{3}}{\dot{\psi}} \\
y_{G c} & =\frac{v_{G x}}{\dot{\psi}} .
\end{aligned}
$$

Remark 1: The coordinate $y_{G c}$ can reach infinite values if the yaw rate $\dot{\psi}=0$, namely, when the robot runs along a perfect straight line under $\omega_{L}=\omega_{R}$. However, the other coordinates $y_{l c}, y_{r c}$, and the $S$ value are bounded. This observation can be explained by the fact that both the numerators and the denominators in (7) and (9a) are simultaneously infinitesimal when the yaw rate $\dot{\psi}$ is close to zero [8]. Therefore, the $y_{l c}, y_{r c}$, and $S$ values are well defined at a finite distance when the yaw rate is zero.

\section{B. IMU Kinematic Motion Equations}

Let $\quad \mathbf{P}_{I}(t)=\left[X_{I}(t) Y_{I}(t) Z_{I}(t)\right]^{T} \in \mathbb{R}^{3} \quad$ and $\quad \mathbf{V}_{I}(t)=$ $\left[V_{x}(t) V_{y}(t) V_{z}(t)\right]^{T} \in \mathbb{R}^{3}$ denote the position and velocity vectors of the IMU in $\mathcal{I}$, respectively. We denote the IMU 
acceleration and angular rate measurements in $\mathcal{B}$ as $\mathbf{A}_{B}=$ $\left[\begin{array}{lll}a_{B x} & a_{B y} & a_{B z}\end{array}\right]^{T} \in \mathbb{R}^{3}$ and $\boldsymbol{\omega}_{B}=\left[\begin{array}{lll}\omega_{B x} & \omega_{B y} & \omega_{B z}\end{array}\right]^{T} \in \mathbb{R}^{3}$, respectively. We obtain the following kinematic motion equations for the IMU [21], [22]:

$$
\begin{aligned}
\dot{\mathbf{P}}_{I} & =\mathbf{V}_{I} \\
\dot{\mathbf{V}}_{I} & =C_{B}^{I} \mathbf{A}_{B}+\mathbf{G} \\
\dot{\phi} & =\omega_{B x}+s_{\phi} \tan \theta \omega_{B y}+c_{\phi} \tan \theta \omega_{B z} \\
\dot{\theta} & =c_{\phi} \omega_{B y}-s_{\phi} \omega_{B z} \\
\dot{\psi} & =\frac{s_{\phi}}{c_{\theta}} \omega_{B y}+\frac{c_{\phi}}{c_{\theta}} \omega_{B z}
\end{aligned}
$$

where $\mathbf{G}=\left[\begin{array}{lll}0 & 0 & -g\end{array}\right]^{T}$, with $g$ being the gravitational constant.

The kinematic models given by (10a) and (10b) describe the relationship between the position vector $\mathbf{P}_{I}$ and the velocity vector $\mathbf{V}_{I}$ in the inertial frame $\mathcal{I}$ and the acceleration measurements $\mathbf{A}_{B}$ in the body frame $\mathcal{B}$. The kinematic models given by (10c)-(10e) describe the relationship between the attitude vector $\Theta$ and the IMU gyro measurements $\boldsymbol{\omega}_{B}$ in $\mathcal{B}$.

\section{Velocity Constraints}

We consider the robot velocity estimates and constraints in the body frame $\mathcal{B}$. Since the robot's four wheels always contact the ground and the IMU is fixed on the robot platform, it is straightforward to obtain the following velocity constraint for the IMU device in the $z$-axis direction:

$$
v_{B z}=0 .
$$

For the IMU longitudinal velocity $v_{B x}$, there is no direct measurement and we obtain an estimate based on the robot's kinematics as follows. Using the definition of the slip in (6), we have $v_{L x}=v_{1 x}=v_{2 x}=\left(1-\lambda_{L}\right) r \omega_{L}$ and $v_{R x}=v_{3 x}=$ $v_{4 x}=\left(1-\lambda_{R}\right) r \omega_{R}$. From (3)-(5), using the wheel encoder reading and the slip estimation, we obtain the longitudinal velocity $v_{B x}$ as

$$
v_{B x}=\frac{r}{2}\left[\left(\omega_{L}+\omega_{R}\right)-\left(\lambda_{L} \omega_{L}+\lambda_{R} \omega_{R}\right)\right]-y_{M} \dot{\psi} .
$$

Since the calculation of wheel slips $\lambda_{L}$ and $\lambda_{R}$ depends on the values of the wheel translational velocity estimates, we need to enforce a bound on slips $\lambda_{L}$ and $\lambda_{R}$ in (12) to ensure a convergent EKF design.

Note that if $\omega_{L} \leq \omega_{R}$, the robot turns left, and the wheel slips satisfy $\lambda_{L} \leq 0$ and $\lambda_{R} \geq 0$. Similarly, we obtain that if $\omega_{L}>\omega_{R}$, then $\lambda_{L}>0$ and $\lambda_{R}<0$. Therefore, we modify (12) as

$$
v_{B x}=\frac{r}{2}\left[\left(\omega_{L}+\omega_{R}\right)-\left(\lambda_{L}^{\prime} \omega_{L}+\lambda_{R}^{\prime} \omega_{R}\right)\right]-y_{M} \dot{\psi}
$$

where

$\lambda_{L}^{\prime}\left(\lambda_{R}^{\prime}\right)= \begin{cases}\max \left\{\lambda_{L}\left(\lambda_{R}\right), 0\right\}, & \text { if } \omega_{L} \geq \omega_{R}\left(\omega_{L} \leq \omega_{R}\right) \\ \min \left\{\lambda_{L}\left(\lambda_{R}\right), 0\right\}, & \text { if } \omega_{L}<\omega_{R}\left(\omega_{L}>\omega_{R}\right) .\end{cases}$

For the lateral velocity $v_{B y}$, from (5) and (7), we obtain

$$
v_{B y}=\left(x_{M}-S\right) \dot{\psi} .
$$

In order to obtain the lateral velocity, we have to estimate the value of $S$. We take an empirical approach to estimate $S$ values, and we will discuss the approach in Section V-B.

\section{Robot LOCALIZATION AND SLIP ESTIMATION}

\section{A. Extended Kalman Filter Design}

With the kinematic models and velocity constraints and estimates, we are now ready to design a kinematic-model-based robot positioning and wheel slip-estimation scheme.

The IMU velocities in frame $\mathcal{B}$ are considered as the measurements $\mathbf{y}(t) \in \mathbb{R}^{3}$, namely

$$
\mathbf{y}(t)=\mathbf{h}\left(\mathbf{v}_{B}\right):=\left[\begin{array}{l}
v_{B x} \\
v_{B y} \\
v_{B z}
\end{array}\right]=\left(C_{B}^{I}\right)^{T} \mathbf{V}_{I} .
$$

Considering the wheel encoder measurement noise and ground topography, we modify (16) and rewrite in discrete-time form as

$$
\mathbf{y}(k)=\mathbf{h}\left(\mathbf{v}_{B}(k)\right)+\mathbf{w}(k)
$$

where the noise signal $\mathbf{w}$ represents the estimation variations that are defined later in this section.

We define the state variable

$$
\mathbf{X}(t):=\left[\begin{array}{lll}
\mathbf{P}_{I}^{T}(t) & \mathbf{V}_{I}^{T}(t) & \boldsymbol{\Theta}^{T}(t)
\end{array}\right]^{T} \in \mathbb{R}^{9}
$$

and rewrite the kinematics (10) in a discrete-time form as

$$
\mathbf{X}(k)=\mathbf{X}(k-1)+\Delta T \mathbf{f}(\mathbf{X}(k-1), \mathbf{u}(k-1))
$$

where $\mathbf{u}(k):=\left[\mathbf{A}_{B}^{T}(k) \boldsymbol{\omega}_{B}^{T}(k)\right]^{T}$ is the IMU measurements at the $k$ th sampling time, and $\Delta T$ is the data-sampling period. The function $\mathbf{f}(\mathbf{X}(k), \mathbf{u}(k))$ is given in (10) as

$$
\mathbf{f}(\mathbf{X}(k), \mathbf{u}(k))=\left[\begin{array}{c}
\mathbf{f}_{P} \\
\mathbf{f}_{V} \\
\mathbf{f}_{\Theta}
\end{array}\right]:=\left[\begin{array}{c}
\mathbf{V}_{I}(k) \\
C_{B}^{I}(k) \mathbf{A}_{B}(k)+\mathbf{G} \\
\mathbf{f}_{\Theta}\left(\boldsymbol{\Theta}(k), \boldsymbol{\omega}_{B}(k)\right)
\end{array}\right]
$$

where $\mathbf{f}_{P}=\mathbf{V}_{I}(k), \mathbf{f}_{V}=C_{B}^{I} \mathbf{A}_{B}(k)+\mathbf{G}$, and

$$
\mathbf{f}_{\Theta}\left(\boldsymbol{\Theta}(k), \boldsymbol{\omega}_{B}(k)\right):=\left[\begin{array}{c}
\omega_{B x}+\tan \theta\left(s_{\phi} \omega_{B y}+c_{\phi} \omega_{B z}\right) \\
c_{\phi} \omega_{B y}-s_{\phi} \omega_{B z} \\
\frac{s_{\phi}}{c_{\theta}} \omega_{B y}+\frac{c_{\phi}}{c_{\theta}} \omega_{B z}
\end{array}\right] .
$$

An EKF design is applied to the systems (16) and (18). For the state dynamics (18), we obtain the Jacobian matrix $\mathbf{F}(k)$ as

$$
\mathbf{F}(k)=\left[\begin{array}{ccc}
\mathbf{I}_{3} & \Delta T \mathbf{I}_{3} & \mathbf{0}_{3} \\
\mathbf{0}_{3} & \mathbf{I}_{3} & \Delta T \mathbf{F}_{V}(k) \\
\mathbf{0}_{3} & \mathbf{0}_{3} & \mathbf{I}_{3}+\Delta T \mathbf{F}_{\Theta}(k)
\end{array}\right]
$$

where $\mathbf{I}_{n}$ and $\mathbf{0}_{n}, n \in \mathbb{N}$, are the $n \times n$ identity and zero matrices, respectively. In (20), we use

$$
\left.\frac{\partial \mathbf{f}}{\partial \mathbf{X}}\right|_{\mathbf{X}(k), \mathbf{u}(k)}=\left[\begin{array}{ccc}
\mathbf{0}_{3} & \mathbf{I}_{3} & \mathbf{0}_{3} \\
\mathbf{0}_{3} & \mathbf{0}_{3} & \mathbf{F}_{V}(k) \\
\mathbf{0}_{3} & \mathbf{0}_{3} & \mathbf{F}_{\Theta}(k)
\end{array}\right]
$$


where

$$
\mathbf{F}_{\Theta}:=\frac{\partial \mathbf{f}_{\Theta}}{\partial \Theta}=\left[\begin{array}{ccc}
\dot{\theta} \tan \theta & \frac{\dot{\psi}}{c_{\theta}} & 0 \\
-\dot{\psi} c_{\theta} & 0 & 0 \\
\frac{\dot{\theta}}{c_{\theta}} & \dot{\psi} \tan \theta & 0
\end{array}\right]
$$

and matrix $\mathbf{F}_{V}$ is given by (22), shown at the bottom of the page. In (22), $\left(C_{B}^{I}\right)_{i j}, i, j=1,2,3$ represents the $i$ th row and $j$ th column component of matrix $C_{B}^{I}$. A detailed calculation of the partial derivatives is listed in the Appendix.

The Jacobian matrix $\mathbf{H}(k)$ for the outputs (16) is calculated as

$$
\mathbf{H}(k)=\left.\frac{\partial \mathbf{h}}{\partial \mathbf{X}}\right|_{\mathbf{X}(k)}=\left[\begin{array}{lll}
\mathbf{0}_{3} & \mathbf{H}_{V}(k) & \mathbf{H}_{\Theta}(k)
\end{array}\right]
$$

where

$$
\mathbf{H}_{V}(k):=\frac{\partial \mathbf{h}\left(\mathbf{v}_{B}\right)}{\partial \mathbf{V}_{I}}=\left(C_{B}^{I}(k)\right)^{T}
$$

and matrix $\mathbf{H}_{\Theta}$ is given by (25), shown at the bottom of the page.

We assume that output measurement noises $\mathbf{w}(k)$ are independent and white, i.e., $\mathbf{w}(k) \sim \mathcal{N}(0, \mathbf{R})$. For the covariance matrix $\mathbf{R}=\operatorname{diag}\left(\sigma_{1}^{2}, \sigma_{2}^{2}, \sigma_{3}^{2}\right) \in \mathbb{R}^{3 \times 3}, \sigma_{1}^{2}=r^{2} \sigma_{e n}^{2}+x_{M}^{2} \sigma_{\dot{\psi}}^{2}$ is the variance for the longitudinal velocity $v_{B x}$ estimate in (13), where $\sigma_{e n}^{2}$ and $\sigma_{\dot{\psi}}^{2}$ are the variances for the wheel encoder measurement and the yaw rate measurement, respectively. $\sigma_{2}^{2}$ is the variance for the lateral velocity estimate $v_{B y}$ in (15). $\sigma_{3}^{2}$ is the variance for the ground surface topography.

The EKF implementation for the systems (17) and (18) can be written as a prediction step $(\hat{\mathbf{X}}(k \mid k-1))$ and a correction step $(\hat{\mathbf{X}}(k \mid k))$ recursively as follows:

$$
\begin{aligned}
\hat{\mathbf{X}}(k \mid k-1)= & \hat{\mathbf{X}}(k-1 \mid k-1) \\
& +\Delta T \mathbf{f}(\hat{\mathbf{X}}(k-1 \mid k-1), \mathbf{u}(k-1)) \\
\mathbf{P}(k \mid k-1)= & \mathbf{F}(k) \mathbf{P}(k \mid k-1) \mathbf{F}^{T}(k)+\mathbf{Q}(k) \\
\hat{\mathbf{X}}(k \mid k)= & \hat{\mathbf{X}}(k \mid k-1) \\
& +\mathbf{W}(k)[\mathbf{y}(k)-\mathbf{H}(k) \hat{\mathbf{X}}(k \mid k-1)] \\
\mathbf{W}(k)= & \mathbf{P}(k \mid k-1) \mathbf{H}^{T}(k) \mathbf{S}^{-1}(k) \\
\mathbf{S}(k)= & \mathbf{H}(k) \mathbf{P}(k \mid k-1) \mathbf{H}^{T}(k)+\mathbf{R} \\
\mathbf{P}(k \mid k)= & \left(\mathbf{I}_{9}-\mathbf{W}(k) \mathbf{H}(k)\right) \mathbf{P}(k \mid k-1) \\
& \times\left(\mathbf{I}_{9}-\mathbf{W}(k) \mathbf{H}(k)\right)^{T}+\mathbf{W}(k) \mathbf{R W}^{T}(k) .
\end{aligned}
$$

In (26b), the symmetric positive-definite matrix $\mathbf{Q}$ is used as a tuning parameter for the EKF performance [25].

The convergence of the EKF design is directly related to the robot motion of the robot. We use the longitudinal velocity calculation in (13) by constraining the slip range in (14). Such a constrained slip calculation helps the EKF design by constraining the initial estimation errors when the wheel slips change significantly during robot motion.

In the EKF design, we do not consider the IMU noise models for several reasons. First, for a clarity of analysis purpose, we neglect the noise models so that we can focus on how to utilize the robot modeling and analysis information for robot position and wheel slip estimation. Second, we have implemented IMU noise models, such as those in [19], in experiments. For a shorttime robot motion, the improved estimation accuracy with the noise models is limited.

\section{B. Velocity-Estimation Error Analysis}

Assuming that the robot attitude $\Theta$ is known, we obtain that the estimates of the velocity given by EKF design are observable and that the EKF could be convergent. In this section, we analyze the velocity-estimation error given a bounded attitude estimation.

Let $\hat{\mathbf{V}}_{I}$ denote the estimated velocity under the estimated attitude $\hat{\boldsymbol{\Theta}}$. From (10b), we obtain

$$
\dot{\hat{\mathbf{V}}}_{I}=\hat{C}_{B}^{I} \mathbf{A}_{B}+\mathbf{G}
$$

where $\hat{C}_{B}^{I}:=C_{B}^{I}(\hat{\boldsymbol{\Theta}})$. The estimated errors for velocity and attitude are defined as

$$
\mathbf{e}_{V}:=\mathbf{V}_{I}-\hat{\mathbf{V}}_{I}, \quad \mathbf{e}_{\Theta}:=\boldsymbol{\Theta}-\hat{\boldsymbol{\Theta}}=\left[\begin{array}{lll}
\Delta \phi & \Delta \theta & \Delta \psi
\end{array}\right]^{T}
$$

respectively. Here, $\Delta \phi:=\phi-\hat{\phi}, \Delta \theta:=\theta-\hat{\theta}$, and $\Delta \psi:=$ $\psi-\hat{\psi}$ are the estimation errors for roll, pitch, and yaw angles, respectively. We assume the estimated attitude error $\mathbf{e}_{\Theta}$ is bounded. From (10b) and (27), we obtain the error dynamics

$$
\dot{\mathbf{e}}_{V}=\Delta C_{B}^{I} \mathbf{A}_{B}
$$

where $\Delta C_{B}^{I}:=C_{B}^{I}-\hat{C}_{B}^{I}$. Using Taylor expansion around $\Theta$, we approximate $\Delta C_{B}^{I}$ as

$$
\Delta C_{B}^{I}=-\left(\frac{\partial C_{B}^{I}}{\partial \phi} \Delta \phi+\frac{\partial C_{B}^{I}}{\partial \theta} \Delta \theta+\frac{\partial C_{B}^{I}}{\partial \psi} \Delta \psi\right)
$$

$$
\mathbf{F}_{V}:=\frac{\partial \mathbf{f}_{V}}{\partial \boldsymbol{\Theta}}=\left[\frac{\partial C_{B}^{I}}{\partial \phi} \mathbf{A}_{B} \frac{\partial C_{B}^{I}}{\partial \theta} \mathbf{A}_{B} \frac{\partial C_{B}^{I}}{\partial \psi} \mathbf{A}_{B}\right]=\left[\begin{array}{ccc}
\left(C_{B}^{I}\right)_{13} a_{B y}-\left(C_{B}^{I}\right)_{12} a_{B z} & c_{\psi}\left(\dot{V}_{z}+g\right) & \dot{V}_{y} \\
\left(C_{B}^{I}\right)_{23} a_{B y}-\left(C_{B}^{I}\right)_{22} a_{B z} & s_{\psi}\left(\dot{V}_{z}+g\right) \\
\left(C_{B}^{I}\right)_{33} a_{B y}-\left(C_{B}^{I}\right)_{32} a_{B z} & -c_{\theta} a_{B x}-s_{\theta} s_{\phi} a_{B y}-c_{\phi} s_{\theta} a_{B z} & 0
\end{array}\right]
$$

$$
\mathbf{H}_{\Theta}:=\frac{\partial \mathbf{h}\left(\mathbf{v}_{B}\right)}{\partial \boldsymbol{\Theta}}=\frac{\partial}{\partial \boldsymbol{\Theta}}\left(\left(C_{B}^{I}\right)^{T} \mathbf{V}_{I}\right)=\left[\begin{array}{ccc}
0 & -s_{\theta} c_{\psi} V_{x}-s_{\theta} s_{\psi} V_{y}-c_{\theta} V_{z} & \left(C_{B}^{I}\right)_{11} V_{y}-\left(C_{B}^{I}\right)_{21} V_{x} \\
v_{z} & s_{\phi} v_{x} & \left(C_{B}^{I}\right)_{12} V_{y}-\left(C_{B}^{I}\right)_{22} V_{x} \\
-v_{y} & c_{\phi} v_{x} & \left(C_{B}^{I}\right)_{13} V_{y}-\left(C_{B}^{I}\right)_{23} V_{x}
\end{array}\right]
$$


Using the aforementioned calculation and (22), (29) is rewritten as

$$
\dot{\mathbf{e}}_{V}=-\mathbf{F}_{V} \mathbf{e}_{\Theta} .
$$

The estimation error analysis only considers the kinematic relationship (10b) under attitude-estimation errors, namely the prediction step (26a) in the EKF design. We need to incorporate the aforementioned analysis with the correction step (26c). Since the EKF design (26) is presented in a discrete-time form, we also analyze the velocity-estimation errors in the discrete-time form for presentation convenience. We rewrite (28) in the discretetime form as

$$
\begin{aligned}
& \mathbf{e}_{V}(k \mid k-1):=\mathbf{V}_{I}(k)-\hat{\mathbf{V}}_{I}(k \mid k-1) \\
& \mathbf{e}_{\Theta}(k \mid k-1):=\boldsymbol{\Theta}(k)-\hat{\boldsymbol{\Theta}}(k \mid k-1)
\end{aligned}
$$

and define the EKF velocity-estimation error

$$
\mathbf{e}_{V}(k \mid k):=\mathbf{V}_{I}(k)-\hat{\mathbf{V}}_{I}(k \mid k) .
$$

Error dynamics (31) are rewritten in the discrete-time form as

$$
\mathbf{e}_{V}(k \mid k-1)=\mathbf{e}_{V}(k-1 \mid k-2)-\Delta T \mathbf{F}_{V}(k) \mathbf{e}_{\Theta}(k \mid k-1) .
$$

Using the definitions in (32), (33) becomes

$$
\begin{aligned}
\mathbf{e}_{V}(k \mid k) & =\mathbf{e}_{V}(k \mid k-1)-\tilde{\mathbf{V}}_{I}(k) \\
& =\mathbf{e}_{V}(k \mid k-1)-(\tilde{\mathbf{X}}(k))_{4: 6}
\end{aligned}
$$

where $\tilde{\mathbf{V}}_{I}(k):=\hat{\mathbf{V}}_{I}(k \mid k)-\hat{\mathbf{V}}_{I}(k \mid k-1), \tilde{\mathbf{X}}(k):=\hat{\mathbf{X}}(k \mid k)-$ $\hat{\mathbf{X}}(k \mid k-1)$, and operator $(\mathbf{X})_{i: j}, i \geq j$ forms a column vector by taking the $i$ th to $j$ th elements from the column vector $\mathbf{X}$. We denote the EKF correction gain matrix in (26c) as $\mathbf{W}(k):=$ $\left[\begin{array}{lll}\mathbf{W}_{1}^{T}(k) & \mathbf{W}_{2}^{T}(k) & \mathbf{W}_{3}^{T}(k)\end{array}\right]^{T} \in \mathbb{R}^{9 \times 3}$, where $\mathbf{W}_{i}(k) \in \mathbb{R}^{3 \times 3}$, $i=1,2,3$. From (23) and (26c), we have

$$
\begin{aligned}
\tilde{\mathbf{V}}_{I}(k)= & (\mathbf{W}(k)[\mathbf{y}(k)-\mathbf{H}(k) \hat{\mathbf{X}}(k \mid k-1)])_{4: 6} \\
= & (\mathbf{W}(k)[\mathbf{y}(k)-\mathbf{H}(k) \mathbf{X}(k)+\mathbf{H}(k) \tilde{\mathbf{X}}(k)])_{4: 6} \\
= & \mathbf{W}_{2}(k)\left[\mathbf{H}_{V}(k) \mathbf{e}_{V}(k \mid k-1)+\mathbf{H}_{\Theta}(k) \mathbf{e}_{\Theta}(k \mid k-1)\right] \\
& -\mathbf{W}_{2}(k)[\mathbf{y}(k)-\mathbf{H}(k) \mathbf{X}(k)] .
\end{aligned}
$$

We use the first-order approximation of the output $\mathbf{y}(k)$ in the second part of the calculation (36). Substituting (36) into (35), we obtain

$$
\begin{aligned}
\mathbf{e}_{V}(k \mid k)= & {\left[\mathbf{I}_{3}-\mathbf{W}_{2}(k) \mathbf{H}_{V}(k)\right] \mathbf{e}_{V}(k \mid k-1) } \\
& -\mathbf{W}_{2}(k) \mathbf{H}_{\Theta}(k) \mathbf{e}_{\Theta}(k \mid k-1) .
\end{aligned}
$$

The velocity-estimation error dynamics (34) and (37) describe the relationship between the EKF velocity-estimation error $\mathbf{e}_{V}(k \mid k)$ and the attitude-estimation error $\mathbf{e}_{\Theta}(k \mid k-1)$. In most of our examples, the mobile robot runs on flat horizontal ground surfaces, and thus, we can approximate $\phi=\theta=0$. Therefore, using (34) and neglecting the attitude dynamics, the velocityestimation errors $\mathbf{e}_{v}(k \mid k-1):=\left(C_{B}^{I}(k)\right)^{T} \mathbf{e}_{V}(k \mid k-1)$ in frame $\mathcal{B}$ satisfy the following dynamic equations:

$$
\begin{aligned}
& \mathbf{e}_{v}(k \mid k-1) \\
& =\mathbf{e}_{v}(k-1 \mid k-2)-\Delta T\left(C_{B 0}^{I}(k)\right)^{T} \mathbf{F}_{V}(k) \mathbf{e}_{\Theta}(k \mid k-1) \\
& =\mathbf{e}_{v}(k-1 \mid k-2)+\Delta T\left[\begin{array}{c}
a_{B y}(k) \\
-a_{B x}(k) \\
0
\end{array}\right] \Delta \psi(k)
\end{aligned}
$$

where

$$
C_{B 0}^{I}(k):=C_{B}^{I}(0,0, \psi(k))=\left[\begin{array}{ccc}
c_{\psi(k)} & -s_{\psi(k)} & 0 \\
s_{\psi(k)} & c_{\psi(k)} & 0 \\
0 & 0 & 1
\end{array}\right] .
$$

Similarly, the EKF velocity-estimation errors (37) are reduced to

$$
\begin{aligned}
\mathbf{e}_{v}(k \mid k)= & {\left[\mathbf{I}_{3}-\left(C_{B 0}^{I}(k)\right)^{T} \mathbf{W}_{2}(k)\right] \mathbf{e}_{v}(k \mid k-1) } \\
& -\left(C_{B 0}^{I}(k)\right)^{T} \mathbf{W}_{2}(k)\left[\begin{array}{c}
v_{B y}(k) \\
-v_{B x}(k) \\
0
\end{array}\right] \Delta \psi(k) .
\end{aligned}
$$

Models (38) and (39) provide a velocity-estimation error for a given yaw angle estimate error. From (38) and (39), the estimation velocity $\mathbf{e}_{v}(k \mid k)$ can grow even if the attitude-estimation error, such as $\Delta \psi(k)$, is bounded.

\section{EXPERIMENTS}

\section{A. Experimental Systems}

We have built an onboard sensor suite on the skid-steered mobile robot, as shown in Fig. 1. The robot and rubber tire size and robot mass are listed in Table I. The robotic tire is deformable and air-damped. The maximum wheel velocity is around $250 \mathrm{r} / \mathrm{min}$, and therefore, the robot can run as fast as around $2 \mathrm{~m} / \mathrm{s}$. We use a low-cost IMU (model IMU 605) from Motion Sense, Inc. The IMU is located at the center of the robot platform, namely $x_{M}=y_{M}=0 \mathrm{~cm}$, for simple calculations. The optical wheel encoder feedback and four-wheel motion control are implemented on a real-time operating system that was developed at the Netbot Laboratory at Texas A\&M University. The control system has a two-level hierarchy: The control algorithm and the EKF design are located in the onboard laptop system, while the PID-based motor control is located at the low-level real-time operating system. The onboard real-time operating system receives the commanded wheel velocity values at a frequency of $20 \mathrm{~Hz}$, and the PID-based motor controller is run at a frequency of $1 \mathrm{KHz}$. The EKF-based positioning and wheel slip estimation scheme is updated at a frequency of 125 $\mathrm{Hz}$, which is also the update frequency of the IMU device.

All experiments were conducted at the Texas A\&M University campus. We have developed a computer-vision-based positioning system to provide the robot's absolute position and yaw angle information in all experiments. A similar system development can be found in [26]. The vision-based velocity and slip calculations are then obtained from the numerical differentiation of the position information. The high-resolution camera system was set up at a fourth-floor location and was calibrated 
TABLE I

ROBOT AND TIRE PARAMETERS

\begin{tabular}{|c|c|c|c|c|}
\hline \hline$L(\mathrm{~cm})$ & $W(\mathrm{~cm})$ & Robot mass $(\mathrm{kg})$ & Tire radius $(\mathrm{cm})$ & Tire width $(\mathrm{cm})$ \\
\hline 36.5 & 41.9 & 10.4 & 8.6 & 9.5 \\
\hline \hline
\end{tabular}

by four landmarks on the ground. These landmarks were formed as a $4 \mathrm{~m} \times 4 \mathrm{~m}$ square. The resolution of the vision positioning system was $1.49 \mathrm{~cm}$.

Between the robot onboard control system and the vision system computer, a timing-synchronization mechanism was set up using wireless communication to correspond the onboard motion-estimation system with the vision-based positioning system. The output of the computer vision-based positioning system only served as a ground truth to compare and validate the proposed robot positioning and wheel slip-estimation scheme.

\section{B. Estimate of the IMU Lateral Velocity Variable $S$}

We developed an empirical model of the lateral velocity variable $S$ as in (15). In what follows first, we consider the dependency of $S$ on ground surface conditions. With the hardware setup that have been discussed in Section V-A, we calculate $S$ using the computer-vision-based positioning system. Fig. 3(a) shows robot trajectories on three different ground surfaces, namely concrete, tile, and concrete with a layer of sand, with constant wheel velocities $\omega_{L} / \omega_{R}=140 / 60 \mathrm{r} / \mathrm{min}$. Fig. 3(b) shows the calculated values of $S$ on these three ground surfaces. It is observed that the value of $S$ does not change significantly under various ground surface conditions. Similar conclusions based on experimental study are also reported in [8] and [15] for tracked vehicles on various ground surfaces.

We then keep the robot motion under the nonsliding condition and estimate the value of $S$ with various wheel velocity combinations. To facilitate the following discussion, we introduce a nondimensional variable $\gamma$ as the ratio of difference and sum of left- and right-side's wheel center linear velocities, namely

$$
\gamma:=\frac{v_{L x}-v_{R x}}{v_{L x}+v_{R x}}=\frac{\left(1-\lambda_{L}\right)-\left(1-\lambda_{R}\right) \kappa_{\omega}}{\left(1-\lambda_{L}\right)+\left(1-\lambda_{R}\right) \kappa_{\omega}}
$$

where $\kappa_{\omega}=\omega_{R} / \omega_{L}$. The value of $S$ is considered as a function of variable $\gamma$. If $\omega_{L}=\omega_{R}$, then $\gamma=0$, and $S$ is a finite value. If $\omega_{L}=-\omega_{R}$, then $\gamma \rightarrow \infty$, and $S=0$ since the robot is rotating about $G$ without any translational motion. Therefore, we approximate the function $S$ in (15) by $\hat{S}$ as follows:

$$
\hat{S}:=\hat{S}(\gamma)=\frac{a_{1}}{a_{2}|\gamma|+a_{3}}
$$

where coefficients $a_{i}>0, i=1,2,3$ are determined by a curve fit of the experimental data.

We ran 11 sets of experiments with various wheel velocity combinations on both concrete and sand road surfaces. Fig. 4 shows the experimental data and the data-fitted curve. Numerical values of parameters $a_{1}=2.148 \mathrm{~cm}, a_{2}=0.249$, and $a_{3}=0.039$ were obtained using a nonlinear least-square algorithm for the function given in (41). Subsequently, we used the estimate of $S(\gamma)$ given in (41) to approximate the lateral velocity $v_{B y}$ in (15).

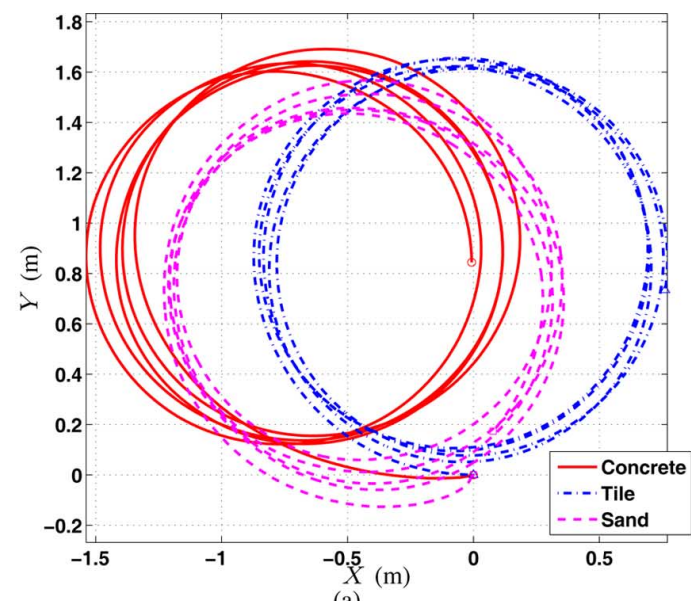

(a)

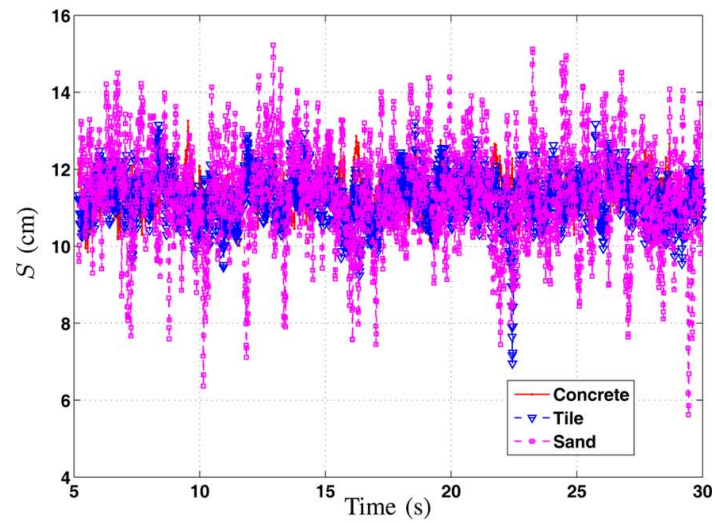

(b)

Fig. 3. (a) Robot trajectories with $\omega_{L}=140 \mathrm{r} / \mathrm{min}$ and $\omega_{R}=60 \mathrm{r} / \mathrm{min}$ on three different ground surfaces. (b) Calculated values of $S$.

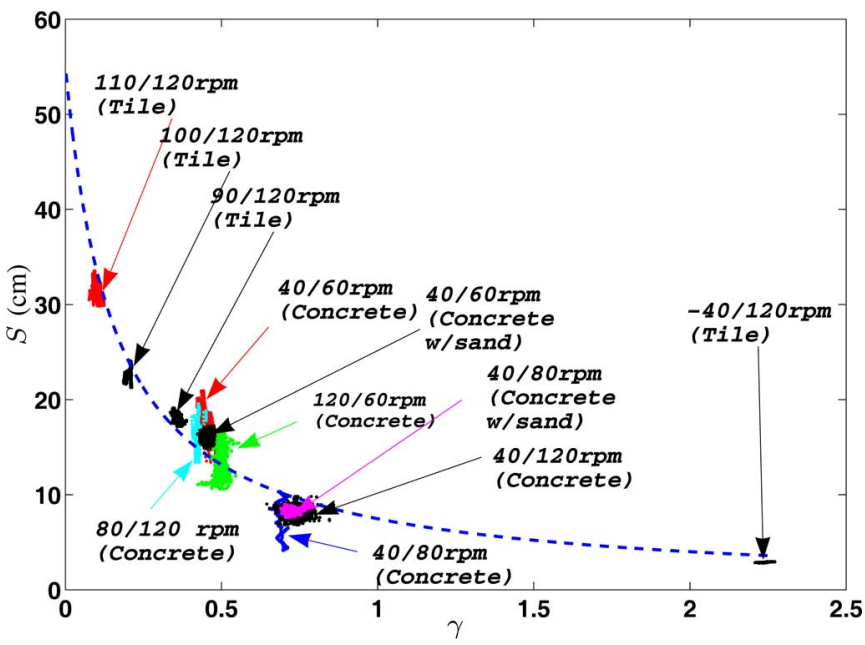

Fig. 4. Experimental data (through the computer-vision-based positioning system) and data-fitted curve (dashed line) by (41) for variable $S$.

Remark 2: Note that in the experiments shown in Fig. 4, the robot wheels do not slide on the ground surface. If wheels are sliding on ground surfaces, such as in sand or on icy surfaces, the calculation of $S$ will be different from (41). Therefore, we can use the $S$-value estimation in (41) as an indicator to detect whether the wheels slide on surfaces. In [3], some experiments have demonstrated such an observation. 


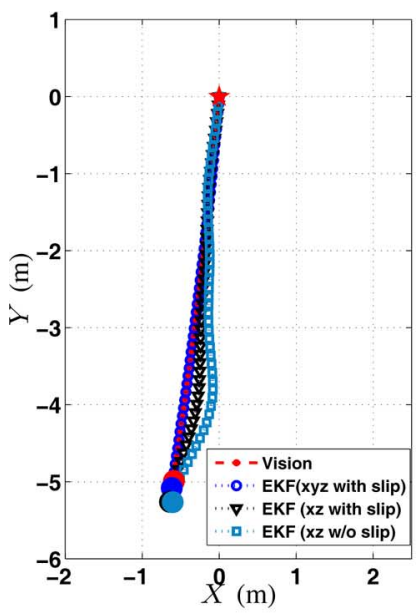

(a)

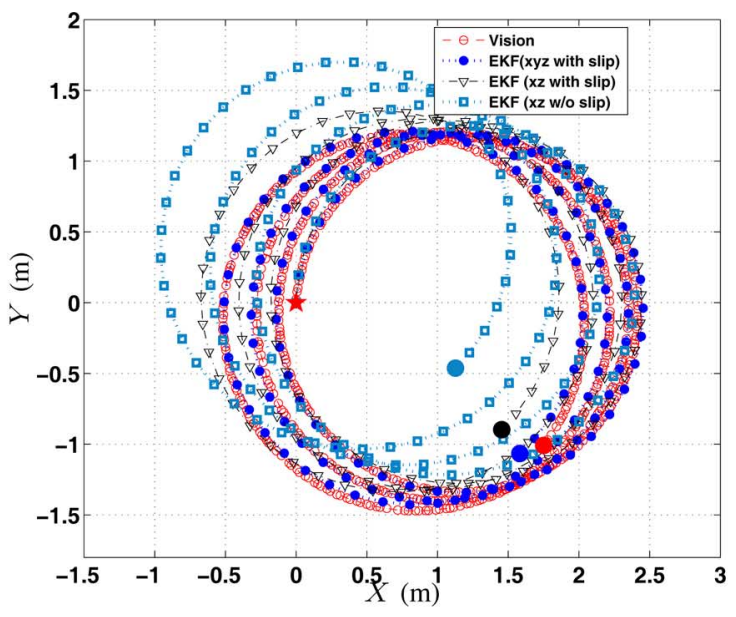

(b)

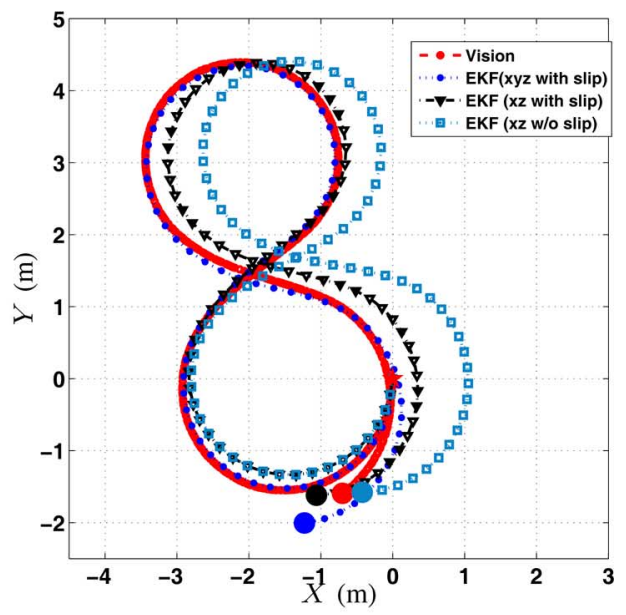

(c)

Fig. 5. EKF-based $X Y$ trajectory estimation for three different shape trajectories. (a) Straight line. (b) Circle. (c) "8" shape. In each trajectory, the EKF-based estimation scheme uses 1$)$ all three $(x y z)$ velocity constraints and estimates with the wheel slip consideration; 2$)$ only two $(x z)$ velocity constraints and estimates with the wheel slip consideration; and 3) only two ( $x z)$ velocity constraints and estimates without the wheel slip consideration. In the figure, the robot starts at the origin (marked as a star symbol) and stops at the symbol marked as a large solid circle.

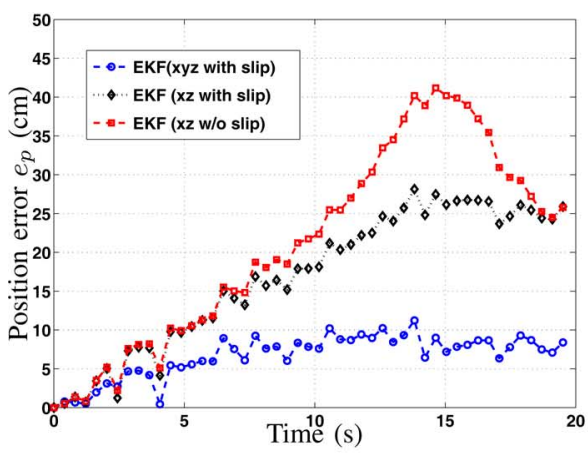

(a)

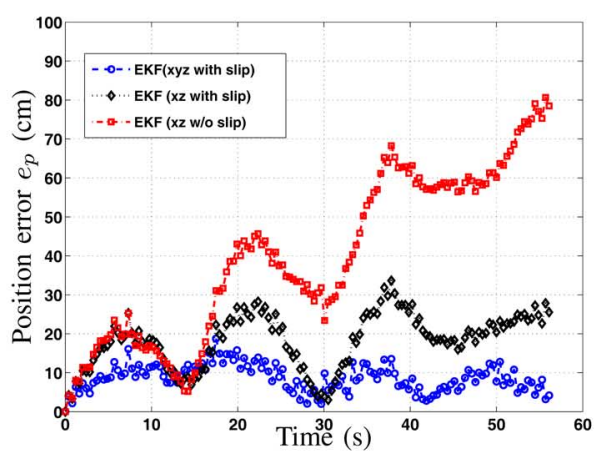

(b)

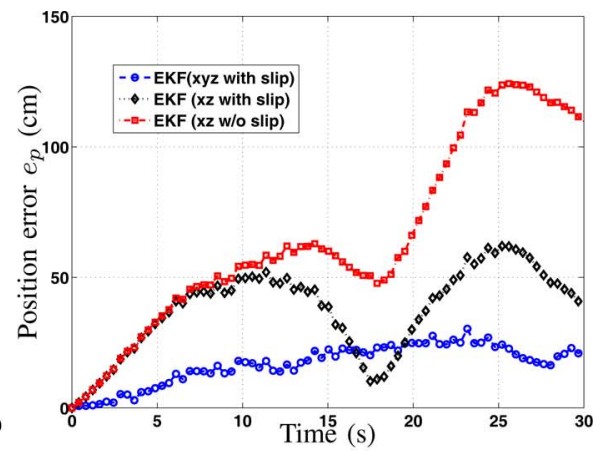

(c)

Fig. 6. EKF-based $X Y$ trajectory estimation position errors $e_{p}$ for three different shape trajectories. (a) Straight line. (b) Circle. (c) "8" shape.

\section{Localization Results}

We present the experimental results where the skid-steered robot runs mainly on a flat concrete surface. Three different trajectory examples are presented here: a straight line, a circle, and an " 8 "-shape trajectory. We chose these three different trajectories to demonstrate the effectiveness of the modeling and motion-estimation schemes.

In Fig. 5, we show the robot positioning results for the three trajectories. For the straight-line trajectory, the robot runs under a constant wheel velocity combination of $\omega_{L}=\omega_{R}=30 \mathrm{r} / \mathrm{min}$. For the circle trajectory, the robot runs under a constant wheel velocity combination of $\omega_{L} / \omega_{R}=80 / 40 \mathrm{r} / \mathrm{min}$. For the " 8 "-shape trajectory, the robot starts with a constant wheel velocity combination of $\omega_{L} / \omega_{R}=80 / 40 \mathrm{r} / \mathrm{min}$ for about $8 \mathrm{~s}$, and then, each side's two wheels simultaneously change their angular velocities by a constant rate of $10 \mathrm{r} /(\mathrm{min} \cdot \mathrm{s})$ until their velocity combination reaches $\omega_{L} / \omega_{R}=40 / 80 \mathrm{r} / \mathrm{min}$. Then, the wheel velocities repeat such a pattern to form the " 8 "-shape trajectory.

Fig. 5 also shows the comparison results of the robot position estimation under three different schemes. These schemes use three different sets of measurements: 1) both the verti- cal velocity $v_{B z}$ constraint and the longitudinal velocity estimate without considering the wheel slip; 2) both the vertical velocity constraint and longitudinal velocity estimate with the wheel slip feedback (13); and 3) all 3-D velocity constraints and estimates with the wheel slip feedback. Here, we do not compare the positioning results by directly integrating the acceleration measurements since such integration results drift significantly away from the true trajectory (see [22] for such comparison examples).

All three types of trajectories show consistent results. From Fig. 5, we observe that the first scheme has a large error (square line). By considering the wheel slip estimation, the localization results improve significantly (triangle line). Finally, if we consider all 3-D velocity constraints and estimations with the slip estimation (dot line), the estimated trajectory is close to the true trajectory. The estimated position errors shown in Fig. 6 further clarify and validate such an observation. In Fig. 6, we take the vision-based positioning system as the true measurements and the estimated position errors $e_{p}$ are defined as the distance differences between the EKF-based position estimates and the vision-based measurements. 


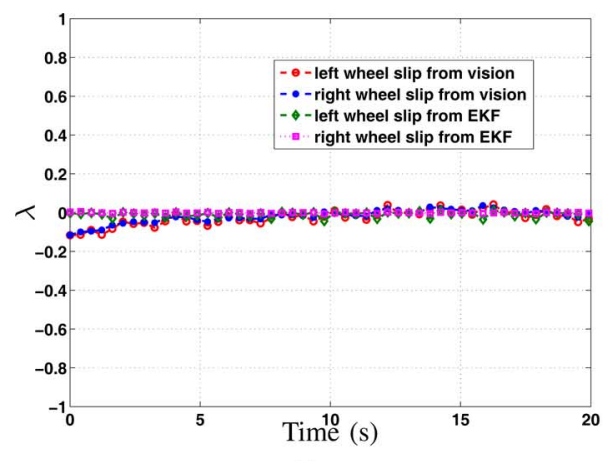

(a)

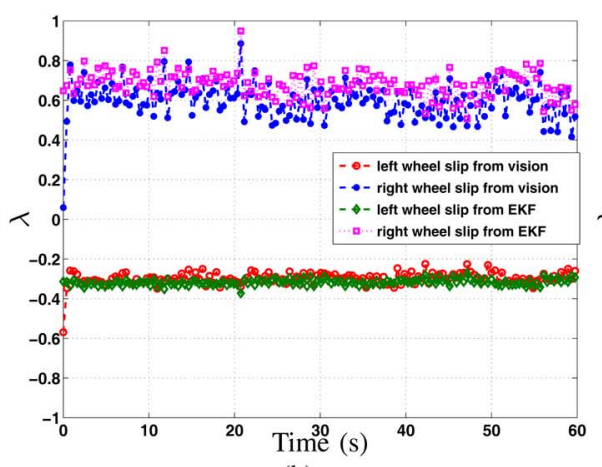

(b)

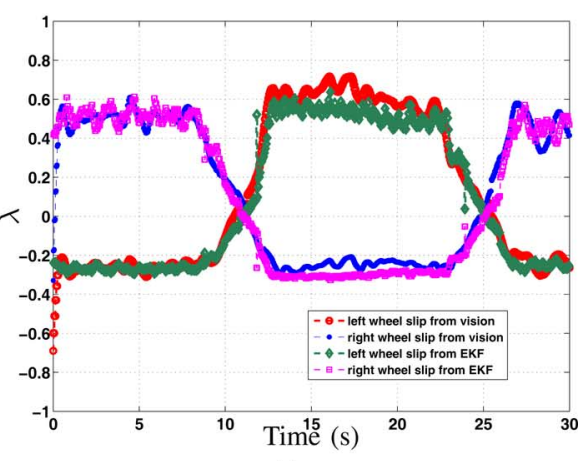

(c)

Fig. 7. Estimated wheel slip information using EKF-based motion-estimation and computer-vision-based positioning schemes under various motion trajectories. (a) Straight line. (b) Circle. (c) "8" shape.

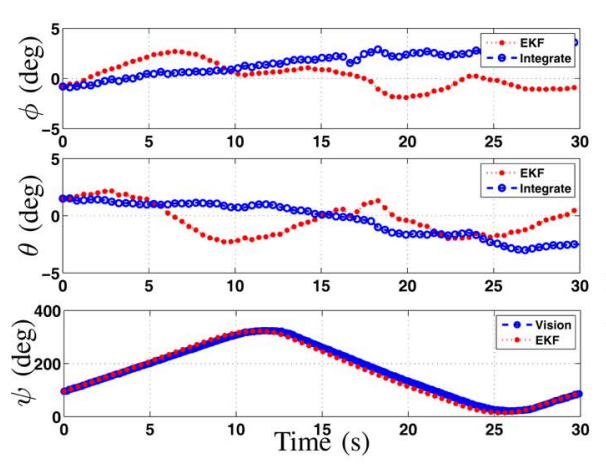

(a)

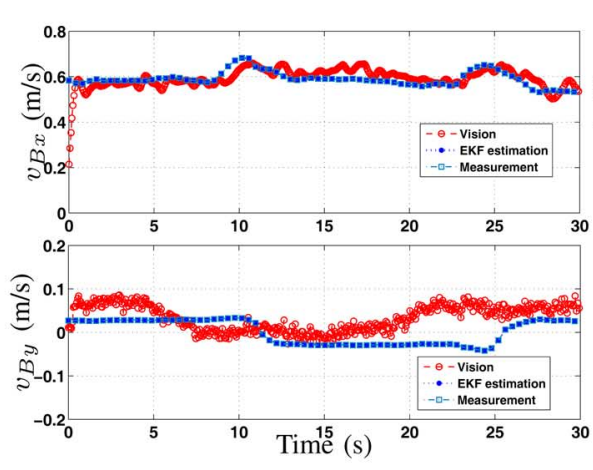

(b)

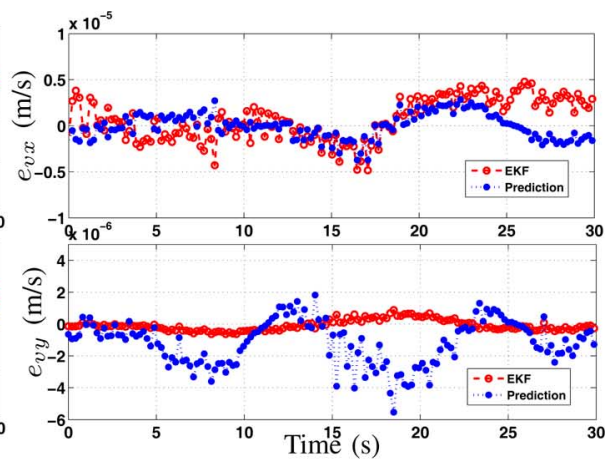

(c)

Fig. 8. Performance of the EKF design for the "8"-shape trajectory. (a) EKF-based robot attitude angle estimates. (b) EKF-based robot velocity estimates in frame $\mathcal{B}$. (c) EKF-based velocity-estimation errors based on the EKF scheme and analytical prediction.

We compare the EKF-based slip estimation under the three trajectories. Fig. 7 shows the wheel slip comparisons between the EKF-based estimation and vision-based measurements. These trajectories represent a set of typical wheel slip values. For example, if the robot runs at $\omega_{L} / \omega_{R}=30 / 30 \mathrm{r} / \mathrm{min}$, i.e., the straight-line trajectory, then wheel slips are close to zero [see Fig. 7(a)] since less force is needed to drive the robot. For the circle trajectory, the robot needs traction/braking forces to overcome the wheel resistance. For the " 8 "-shape trajectory, the slip values of one side's wheels change signs, as shown in Fig. 7(c). The slip values shown in Fig. 7(c) clearly show that the wheel velocity changes for the "8"-shape trajectory. All of the EKF-based slip estimates shown in Fig. 7 closely match the vision-based slip values.

As we demonstrated in the analysis (31), the velocityestimation errors are closely related to the estimation errors of the attitude. Fig. 8(a) shows the EKF-based attitude angle estimation for the " 8 "-shape trajectory. The top two subfigures in Fig. 8(a) show the comparison estimates of the roll and pitch angles between the EKF-based scheme and the direct integration method. For the roll and pitch angles, the EKF-based estimation performs better than the direct integration results within the $30-\mathrm{s}$ robot running time. This is not surprising since the random walk noise in the gyro measurements has a standard deviation that is proportional to the square root of time [21]. The bottom subfigure in Fig. 8(a) compares the EKF-based yaw angle estimate with the vision-based system measurements.
We show some results for the EKF performance. Fig. 8 shows the robot velocities and the EKF velocity-estimation errors in $\mathcal{B}$ for the " 8 "-shape trajectory. Fig. 8(b) shows comparison results between the EKF-based estimation and the vision-based positioning system measurements. Fig. 8(c) shows the EKF-based velocity-estimation errors. The EKFbased velocity estimates follow the robot's true velocity profiles.

From Fig. 8(a), we know that the robot runs on a fairly flat surface since the roll and pitch angles are close to zero. Therefore, we can use the relationship given in (38) and (39) to predict the velocity-estimation errors under the yaw-angleestimation error $\Delta \psi$. In Fig. 8(c), we compare such predictions (solid-circle line) with the EKF-based estimation errors (emptycircle line), and they match well in the $x$-axis and $y$-axis directions. Fig. 9 shows the $S$-value comparison results for both the circle and the "8"-shape trajectories. For the circle trajectory, the $S$ values remain constant during the motion, while for the "8"-shape trajectory, the $S$ values keep changing under $\gamma$ variations as in (41).

\section{CONCLUSION AND FUtURE WORK}

We presented a kinematic modeling and analysis of skidsteered mobile robots. The kinematic analysis was based on the motion similarity between the skid-steered robot and tracked 


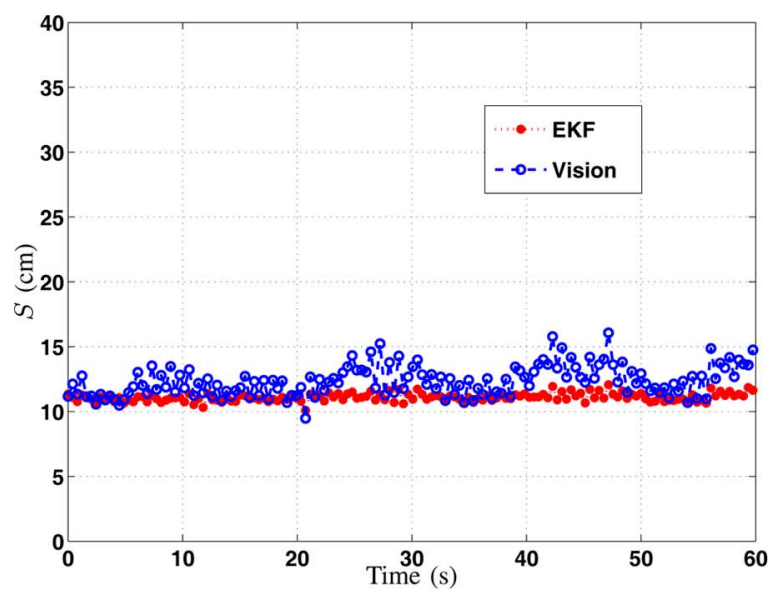

(a)

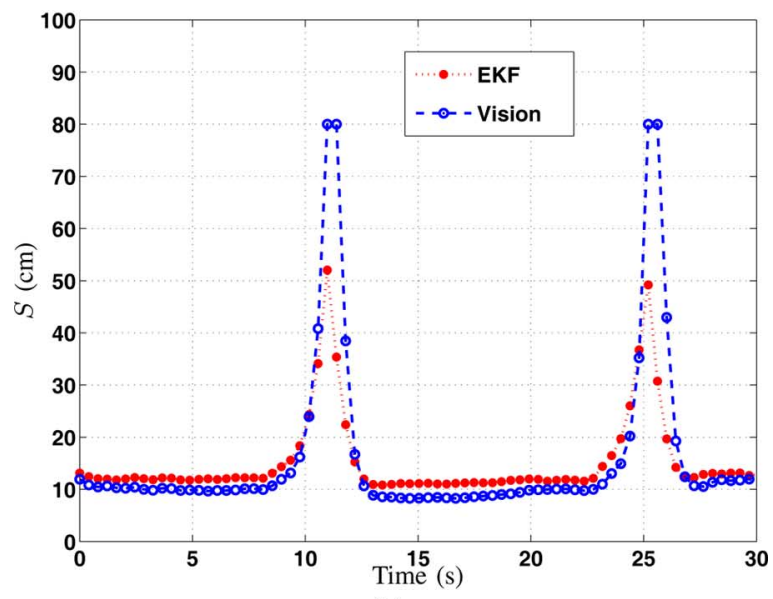

(b)

Fig. 9. $S$-value comparison. (a) Circle trajectory. (b) "8"-shape trajectory.

vehicles. The modeling analyses demonstrated that the location of the instantaneous center of rotation plays an important role for wheel slip and robot velocity estimations. We also presented an EKF-based motion-estimation scheme for the skid-steered robot using only a low-cost strapdown IMU and wheel encoder measurements. The EKF design utilized the "virtual" velocity measurements from the robot kinematic modeling and analysis. The analysis and estimation methodology were tested experimentally on a robotic test bed. The experimental comparison results showed a superior performance for the proposed motion-estimation scheme than those not using the new kinematics-model-based "virtual" velocity measurements.

We are currently conducting several research extensions. A slip-based trajectory controller is under development to be integrated with the slip estimation scheme for robot dynamic and motion stability analysis [3], [12]. Another ongoing research activity is to integrate the "smart tire" sensing information, such as the one in [27], with an onboard computer to develop a new control system that can utilize wheel skidding as an actuation mechanism for a better robot motion performance.

\section{APPENDIX}

From (2), it is straightforward to calculate the derivatives of $C_{B}^{I}$ with respect to the attitude angles as follows:

$$
\begin{aligned}
\frac{\partial C_{B}^{I}}{\partial \phi}= & {\left[\begin{array}{lll}
0 & \left(C_{B}^{I}\right)_{13} & -\left(C_{B}^{I}\right)_{12} \\
0 & \left(C_{B}^{I}\right)_{23} & -\left(C_{B}^{I}\right)_{22} \\
0 & \left(C_{B}^{I}\right)_{33} & -\left(C_{B}^{I}\right)_{32}
\end{array}\right] } \\
\frac{\partial C_{B}^{I}}{\partial \theta}= & {\left[\begin{array}{ccc}
c_{\psi}\left(C_{B}^{I}\right)_{31} & c_{\psi}\left(C_{B}^{I}\right)_{32} & c_{\psi}\left(C_{B}^{I}\right)_{33} \\
s_{\psi}\left(C_{B}^{I}\right)_{31} & s_{\psi}\left(C_{B}^{I}\right)_{32} & s_{\psi}\left(C_{B}^{I}\right)_{33} \\
-c_{\theta} & -s_{\theta} s_{\phi} & -s_{\theta} c_{\phi}
\end{array}\right] }
\end{aligned}
$$

and

$$
\frac{\partial C_{B}^{I}}{\partial \psi}=\left[\begin{array}{ccc}
-\left(C_{B}^{I}\right)_{21} & -\left(C_{B}^{I}\right)_{22} & -\left(C_{B}^{I}\right)_{23} \\
\left(C_{B}^{I}\right)_{11} & \left(C_{B}^{I}\right)_{12} & \left(C_{B}^{I}\right)_{13} \\
0 & 0 & 0
\end{array}\right] .
$$

\section{REFERENCES}

[1] A. Angelova, L. Matthies, D. Helmick, and P. Perona, "Slip prediction using visual information," in Proc. Robot.: Sci. Syst., Philadelphia, PA, 2006.

[2] D. M. Helmick, S. I. Roumeliotis, Y. Cheng, D. S. Clouse, M. Bajracharya, and L. H. Matthies, "Slip-compensated path following for planetary exploration rovers," Adv. Robot., vol. 20, no. 11, pp. 1257-1280, 2006.

[3] H. Wang, J. Zhang, J. Yi, D. Song, S. Jayasuriya, and J. Liu, "Modeling and motion stability analysis of skid-steered mobile robots," in Proc. IEEE Int. Conf. Robot. Autom., Kobe, Japan, 2009, pp. 4112-4117.

[4] Z. Song, Y. H. Zweiri, L. D. Seneviratne, and K. Althoefer, "Nonlinear observer for slip estimation of skid-steering vehicles," in Proc. IEEE Int. Conf. Robot. Autom., Orlando, FL, 2006, pp. 1499-1504.

[5] A. T. Le, D. C. Rye, and H. F. Durrant-Whyte, "Estimation of track-soil interactions for autonomous tracked vehicles," in Proc. IEEE Int. Conf. Robot. Autom., Albuquerque, NM, 1997, pp. 1388-1393.

[6] G. Anousaki and K. J. Kyriakopoulos, "A dead-reckoning scheme for skid-steered vehicles in outdoor environments," in Proc. IEEE Int. Conf. Robot. Autom., New Orleans, LA, 2004, pp. 580-585.

[7] G. Anousaki and K. J. Kyriakopoulos, "Simultaneous localization and map building of skid-steered robots," IEEE Robot. Autom. Mag., vol. 14, no. 1, pp. 79-89, Mar. 2007.

[8] J. L. Martínez, A. Mandow, J. Morales, S. Pedraza, and A. García-Cerezo, "Approximating kinematics for tracked mobile robots," Int. J. Robot. Res., vol. 24, no. 10, pp. 867-878, 2005.

[9] A. Mandow, J. L. Martínez, J. Morales, J. L. Blanco, A. García-Cerezo, and J. González, "Experimental kinematics for wheeled skid-steer mobile robots," in Proc. IEEE/RSJ Int. Conf. Intell. Robot. Syst., San Diego, CA, 2007, pp. 1222-1227.

[10] R. Balakrishna and A. Ghosal, "Modeling of slip for wheeled mobile robots," IEEE Trans. Robot. Autom., vol. 11, no. 1, pp. 126-132, Feb. 1995.

[11] D. Wang and C.-B. Low, "Modeling and analysis of skidding and slipping in wheeled mobile robots: Control design perspective," IEEE Trans. Robot., vol. 24, no. 3, pp. 676-687, Jun. 2008.

[12] J. Yi, D. Song, J. Zhang, and Z. Goodwin, "Adaptive trajectory tracking control of skid-steered mobile robots," in Proc. IEEE Int. Conf. Robot. Autom., Rome, Italy, 2007, pp. 2605-2610.

[13] L. Ojeda and J. Borenstein, "Methods for the reduction of odometry errors in over-constrained mobile robots," Auton. Robots, vol. 16, no. 3, pp. 273286, 2004.

[14] L. Ojeda, D. Cruz, G. Reina, and J. Borenstein, "Current-based slippage detection and odometry correction for mobile robots and planetary rovers," IEEE Trans. Robot., vol. 22, no. 2, pp. 366-378, Apr. 2006.

[15] D. Endo, Y. Okada, K. Nagatani, and K. Yoshida, "Path following control for tracked vehicles based on slip-compensating odometry," in Proc. IEEE/RSJ Int. Conf. Intell. Robot. Syst., San Diego, CA, 2007, pp. 28712876.

[16] M. Kitano and M. Kuma, "An analysis of horizontal plane motion of tracked vehicles," J. Terramech., vol. 14, no. 4, pp. 211-225, 1977. 
[17] J. Y. Wong, Theory of Ground Vehicles, 3rd ed. Hoboken, NJ: Wiley, 2001.

[18] J. Y. Wong and C. F. Chiang, "A general theory for skid steering of tracked vehicles on firm ground," in Proc. Inst. Mech. Eng., Part D: J. Automobile Eng., 2001, vol. 215, pp. 343-355.

[19] C. C. Ward and K. Iagnemma, "A dynamic-model-based wheel slip detector for mobile robot on outdoor terrain," IEEE Trans. Robot., vol. 24, no. 4, pp. 821-831, Aug. 2008.

[20] S. Schedling, G. Dissanayake, E. Nebot, and H. Durrant-Whyte, "Slip modelling and aided inertial navigation of an LHD," in Proc. IEEE Int. Conf. Robot. Autom., Albuquerque, NM, 1997, pp. 1904-1909.

[21] S. Sukkarieh, "Low cost, high integrity aided inertial navigation systems for land vehicle applications," Ph.D. dissertation, Dept. Mech. Mechatron. Eng., Univ. Sydney, Sydney, N.S.W, Australia, 2000.

[22] G. Dissanayake, S. Sukkarieh, E. Nebot, and H. Durrant-Whyte, "The aiding of a low-cost strapdown inertial measurement unit using vehicle model constraints for land vehicle applications," IEEE Trans. Robot. Autom., vol. 17, no. 5, pp. 731-747, Oct. 2001.

[23] J. Yi, J. Zhang, D. Song, and S. Jayasuriya, "IMU-based localization and slip estimation for skid-steered mobile robots," in Proc. IEEE/RSJ Int. Conf. Intell. Robot. Syst., San Diego, CA, 2007, pp. 2845-2850.

[24] K. R. Weiss, "Skid-steering," Auto. Eng., pp. 22-25, 1971.

[25] M. Boutayeb and D. Aubry, "A strong tracking extended Kalman observer for nonlinear discrete-time systems," IEEE Trans. Autom. Control, vol. 44, no. 8, pp. 1550-1556, Aug. 1999.

[26] T. D'Orazio, F. P. Lovergine, M. Ianigro, E. Stella, and A. Distante, "Mobile robot position determination using visual landmarks," IEEE Trans. Ind. Electron., vol. 41, no. 6, pp. 654-662, Dec. 1994.

[27] J. Yi, "A piezo-sensor based "smart tire" system for mobile robots and vehicles," IEEE/ASME Trans. Mechatron., vol. 13, no. 1, pp. 95-103, Feb. 2008.

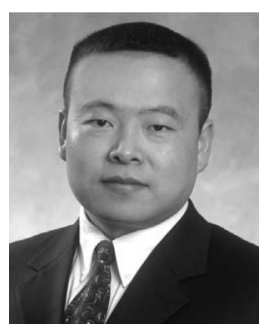

Jingang Yi (S'99-M'02-SM'07) received the B.S. degree in electrical engineering from Zhejiang University, Hangzhou, China, in 1993, the M.Eng. degree in precision instruments from Tsinghua University, Beijing, China, in 1996, and the M.A. degree in mathematics and the Ph.D. degree in mechanical engineering from the University of California, Berkeley, in 2001 and 2002, respectively.

From May 2002 to January 2005, he was with Lam Research Corporation, Fremont, CA, as a Member of the Technical Staff. From January 2005 to December 2006, he was with the Department of Mechanical Engineering, Texas A\&M University, College Station, as a Visiting Assistant Professor. From January 2007 to July 2008, he was an Assistant Professor of mechanical engineering with San Diego State University, San Diego, CA. In August 2008, he joined Rutgers University, Piscataway, NJ, where he is currently an Assistant Professor of mechanical engineering. His current research interests include autonomous robotic systems, dynamic systems and control, intelligent sensing and actuation systems, mechatronics, and automation science and engineering, with applications to semiconductor manufacturing, intelligent transportation, and biomedical systems.

Dr. Yi is a member of American Society of Mechanical Engineers (ASME). $\mathrm{He}$ is a member of the Conference Editorial Board of the IEEE Robotics and Automation Society and ASME Dynamic Systems and Control Division. He is a Guest Editor of the IEEE TRANSACTIONS ON AUtOMATION SCIENCE AND ENGINEERING.

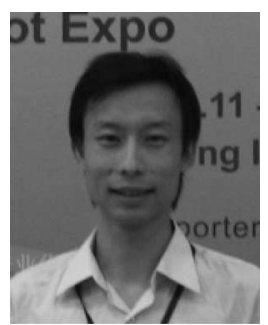

Hongpeng Wang (M'09) received the B.S. degree in physics and the Ph.D. degree in control theory and engineering from Nankai University, Tianjin, China, in 2000 and 2009, respectively.

$\mathrm{He}$ is currently a faculty member with the Institute of Robotics and Automatic Information Systems, Nankai University. His current research interests include autonomous robotic systems, intelligent sensing systems, and geography space information, with applications to intelligent transportation and surveying engineering. During 2007-2008, he was a Visiting Research Fellow with the Department of Computer Science and Engineering, Texas A\&M University, College Station.

Dr. Wang is a member of the IEEE Robotics and Automation Society.

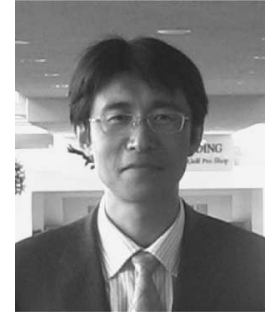

Junjie Zhang (S'08) received the B.S. degree in mechanical engineering and the B.A. degree in Japanese from Dalian University of Technology, Dalian, China, in 2003 and the M.S. degree in mechanical engineering from the University of Michigan, Ann Arbor, in 2005. He is currently working toward the Ph.D. degree with the Department of Mechanical Engineering, Texas A\&M University, College Station.

His current research interests include dynamic systems and control, autonomous robots, cooperative control of multirobot systems, semiconductor manufacturing automation, intelligent maintenance, and mechatronics.

Mr. Zhang is a Student Member of the American Society of Mechanical Engineers and the IEEE Robotics and Automation Society (RAS). He is currently the Student Activities Committee Cochair of the 2009 IEEE RAS Conference.

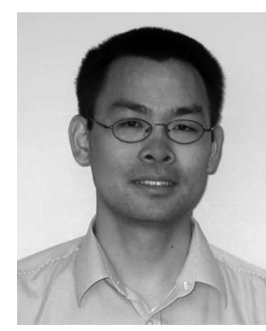

Dezhen Song (S'02-M'04) received the Ph.D. degree in engineering from the University of California, Berkeley, in 2004.

$\mathrm{He}$ is currently an Assistant Professor with Texas A\&M University, College Station. His current research interests include networked robotics, computer vision, optimization, and stochastic modeling.

Dr. Song received the Kayamori Best Paper Award at the 2005 IEEE International Conference on Robotics and Automation. He received the $\mathrm{Na}$ tional Science Foundation CAREER Award in 2007. From 2007 to 2009, he was the Cochair of the IEEE Robotics and Automation Society Technical Committee on Networked Robots. He is an Associate Editor of the IEEE TRANSACTIONS ON ROBOTICS.

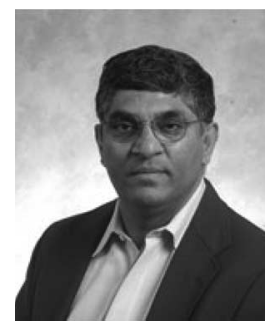

Suhada Jayasuriya (S'81-M'82-SM'02) received the $\mathrm{Ph} . \mathrm{D}$. degree from Wayne State University, Detroit, MI, in 1982.

Until 1987, he was with Michigan State University, East Lansing. Since 1987, he has been with Texas A\&M University, College Station, where he is currently a Professor of mechanical engineering, has been the Kotzebue Professor since 1994, and was the Head of the Department of Mechanical Engineering from 1997 to 2001 . He is currently the Director of the Control Systems Program with the Civil, Mechanical, and Manufacturing Innovation (CMMI) Division, National Science Foundation His current research interests include frequency-domain robust control synthesis, vibration and flow control, nanoprecision motion control, and cooperative control and sensor networks.

Dr. Jayasuriya was elected Fellow of the American Society of Mechanical Engineers (ASME) in 1994. He received the Gustus L. Larson Memorial Award from ASME in 1997 and the Outstanding Investigator Award of the ASME Dynamic Systems and Control Division in 2002. He was the General Chair of the 2005 American Control Conference and the Chairman of the ASME Dynamic Systems and Control Division (2002-2003). During 2003-2008, he was the Editor of the ASME Journal of Dynamic Systems, Measurement, and Control.

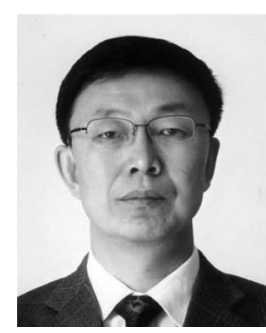

Jingtai Liu (M'09) received the B.S. degree in engineering of industry automation from Tianjin University, Tianjin, China, in 1983, the M.Eng. degree in electrical engineering from Tianjin University in 1986, and the Ph.D. degree in robotics engineering from Nankai University, Tianjin, in 1998.

$\mathrm{He}$ is currently the Director of the Institute of Robotics and Automatic Information System, Nankai University, where he is also a Professor with the Information School. His current research interests include robotics and automatic control, competitive networked robots, wireless sensor networks, automatic information systems, an robotics education for kids. During 2006-2007, he was a Visiting Scholar with the Laboratory for Automation Science and Engineering, University of California, Berkeley.

Dr. Liu was a member of the Industry Robotics Technology Expert Group, which was a High-tech Research and Development Program of China (China 863 Program), during 1997-2001. 